\title{
Adsorption and Desorption of Steroid Hormones by Microplastics in Seawater
}

\author{
Jian $\mathrm{Lu}^{1,2} \cdot$ Jie $\mathrm{Wu}^{1,2} \cdot$ Jun $\mathrm{Wu}^{3} \cdot$ Cui Zhang ${ }^{1,2} \cdot$ Yongming $\mathrm{Luo}{ }^{1}$
}

Received: 1 October 2019 / Accepted: 3 January 2020

(c) Springer Science+Business Media, LLC, part of Springer Nature 2020

\begin{abstract}
This study evaluated the adsorption and desorption of $17 \beta$-estradiol (E2) and 17 $\alpha$-ethynylestradiol (EE2) on microplastics in seawater. The effects of microplastic materials and particle sizes on adsorption of E2 and EE2 were explored. Moreover, effects of salinity, $\mathrm{pH}$, humic acid (HA) concentrations, and initial E2/EE2 concentrations on adsorption were also discussed. Increase in salinity, HA concentration, and initial E2/EE2 concentration would enhance adsorption of E2/EE2 on microplastics. Adsorption capacity of E2/EE2 firstly increased to reach the highest at $\mathrm{pH}$ of 8.0 and then decreased when $\mathrm{pH}$ further increased. Pseudo-second-order kinetics better fitted adsorption data of E2 while pseudo-first-order model yielded better fitting results for EE2. Freundlich isotherm was better to fit the adsorption data of E2 while Langmuir isotherm yielded better fitting results for EE2. Desorption capacity of E2/EE2 on microplastics was over $40 \%$ of its adsorption capacity. This study provides new insights on microplastics and endocrine disrupting chemicals.
\end{abstract}

Keywords Microplastics $\cdot$ Endocrine disrupting chemicals $\cdot$ Adsorption $\cdot$ Desorption $\cdot$ Seawater

Plastics, widely produced and used since the 1940s, are biologically inert and chemically stable to persist in the environment (O'Brine and Tompson 2010). Microplastics, plastic pieces with size $<5.00 \mathrm{~mm}$, possess relatively large specific surface area and high hydrophobic properties to easily adsorb and enrich hydrophobic organic pollutants to threat ecosystems and humans because of easy ingestion by organisms (Mato et al. 2001; Ogata et al. 2009; Zhang et al. 2018). Microplastics have widely existed in various matrices and become an important concern in coastal aquatic environment (Andrady 2011; Barboza and Gimenez 2015).

Endocrine disrupting chemicals (EDCs) will adversely affect the ecosystems and human health even at very low

Jian Lu

jlu@yic.ac.cn

1 CAS Key Laboratory of Coastal Environmental Processes and Ecological Remediation, Yantai Institute of Coastal Zone Research (YIC), Chinese Academy of Sciences (CAS); Shandong Key Laboratory of Coastal Environmental Processes, YICCAS, Yantai 264003, Shandong, China

2 University of Chinese Academy of Sciences, Beijing 100049, China

3 School of Resources and Environmental Engineering, Ludong University, Yantai 264025, Shandong, China concentrations due to feminization effects (Brian et al. 2007). Steroid hormones, kind of endogenous EDCs, can cause feminization of wild fish at $\mathrm{ng} \mathrm{L}^{-1}$ levels (Giulivo et al. 2016). However, information on EDCs adsorbed on microplastics is still limited although these contaminants are easily adsorbed on microplastics due to their strong hydrophobic properties (Wu et al. 2019; Ying et al. 2002). Therefore, it is necessary to study the adsorption-desorption behavior of EDCs on microplastics in the aquatic environment, especially marine/coastal ecosystems. This study performed batch assays to investigate adsorption and desorption of typical steroid hormones including $17 \beta$-estradiol (E2) and $17 \alpha$-ethynylestradiol (EE2) on microplastics in coastal water under different conditions. The final aim of this study is to provide useful information on interfacial behaviors of EDCs and microplastics.

\section{Materials and Methods}

The microplastics with particle size range of $4-5 \mathrm{~mm}$ and relatively smooth surface including polyvinyl chloride (PVC, mean size: $4.7 \mathrm{~mm}$ ), polypropylene ( $\mathrm{PP}$, mean size: $4.51 \mathrm{~mm}$ ), and polyethylene (PE, mean size: $4.85 \mathrm{~mm}$ ) were purchased from LG Company (Korea) while PVC particles 
(size range of $0.10-0.13 \mathrm{~mm}$ and mean size of $0.11 \mathrm{~mm}$ ) with irregular structure and relatively rough surface were obtained from Guangzhou Bofeng Chemical Technology Company (China). E2 and EE2 standards with purity > 97\% were purchased from Tiexiai Chemical Industrial Development Company (Shanghai, China). Acetonitrile and methanol with HPLC grade were obtained from Merck (Germany). Humic acid (HA) was obtained according to Meng et al. (2017). Stock solutions of E2 and EE2 with concentration of $1 \mathrm{~g} \mathrm{~L}^{-1}$ were weekly prepared and stored at $-20^{\circ} \mathrm{C}$. Working solutions were prepared by diluting stock solution with sterile seawater before use. Seawater was acquired from the eastern sea areas of Yantai with $\mathrm{pH}$ of 7.84-8.09 and saltity of $32 \mathrm{~g} \mathrm{~L}^{-1}$. Seawater for experiments was filtered through $0.22 \mu \mathrm{m}$ glass fiber filters (HNTY Company, Haining, China) and used after sterilization.

Conditions including the initial E2/EE2 concentration of $10 \mu \mathrm{g} \mathrm{L}^{-1}$, microplastic concentration of $0.05 \mathrm{~g} \mathrm{~L}^{-1}$, temperature of $25^{\circ} \mathrm{C}$, salinity of $32 \mathrm{~g} \mathrm{~L}^{-1}$, and shaking speed of $170 \mathrm{rpm}$ were set if no specific comments were proposed. Particle sizes of PVC were chosen as 0.11 and $4.7 \mathrm{~mm}$. The effects of different factors including salinity, $\mathrm{pH}, \mathrm{HA}$ concentration, and initial concentrations of EDCs on the adsorption of E2/EE2 by PVC $(0.11 \mathrm{~mm})$ microplastics were investigated. Salinity was set at 16,24 , and $32 \mathrm{~g} \mathrm{~L}^{-1}$ while solution $\mathrm{pH}$ was adjusted as $6,7,8,9$, and 10 . HA concentrations were set as $0,2,5,10$, and $20 \mathrm{mg} \mathrm{L}^{-1}$ while initial concentrations of EDCs were set as 5, 10, 20, 50, and $100 \mu \mathrm{g} \mathrm{L}^{-1}$. All assays were performed in triplicate $(\mathrm{n}=3)$ and the relative standard deviations (RSD) of most of data were below 5\%. The microplastic-free controls were performed to eliminate the disturbance. The one-way ANOVA analysis and Pearson correlation analysis were used to determine difference in effect of various factors on adsorption of E2 and EE2.

Adsorption kinetics of E2/EE2 on PVC $(0.11 \mathrm{~mm})$ microplastics were investigated by collecting samples at $0,1,2$, 6, 10, 24, 36, 48, 72, and 96 h. Desorption kinetics were studied by collecting samples at $0,2,4,8,12,24,36,48$, and $72 \mathrm{~h}$ after adsorption equilibrium. Pseudo-first order and pseudo-second order models were used to describe adsorption and desorption kinetics as the followings ( $\mathrm{Lu}$ et al. 2017):

$\ln \left(q_{e q}-q_{t}\right)=\ln q_{e q}-k_{1} t ; \frac{t}{q_{t}}=\frac{1}{k_{2} q_{e q}^{2}}+\frac{1}{q_{e q}} t$

$q_{e q}=\frac{\left(C_{0}-C_{e q}\right) \times V}{W} ; q_{t}=\frac{\left(C_{0}-C_{t}\right) \times V}{W}$

where $q_{e q}$ and $q_{t}$ are amount of E2/EE2 adsorbed by unit mass microplastics at equilibrium and at time $t$, respectively; $k_{1}$ and $k_{2}$ refer to the rate constant for pseudo-first-order model and pseudo-second-order model, respectively; $C_{0}$, $C_{e q}$, and $C_{t}$ refer to the initial concentration, concentration at equilibrium, and concentration at time $t$ of target antibiotics in liquid phase, respectively; $V$ is the volume of the solution; $W$ is the mass of microplastics.

Adsorption isotherms were investigated under temperature of $15^{\circ} \mathrm{C}(288 \mathrm{~K}), 25^{\circ} \mathrm{C}(298 \mathrm{~K})$, and $35^{\circ} \mathrm{C}(308 \mathrm{~K})$ with E2/EE2 concentrations of 5, 10, 20, 50, and $100 \mu \mathrm{g} \mathrm{L}^{-1}$. The Langmuir model and Freundlich isotherm were used to fit the isotherm data as the followings ( $\mathrm{Lu}$ et al. 2017):

$\frac{1}{q_{e q}}=\frac{1}{k_{L} Q_{m} C_{e q}}+\frac{1}{Q_{m}} ; q_{e q}=k_{F} C_{e q}^{\frac{1}{n}}$

where $k_{L}$ is the surface adsorption equilibrium constant; $Q_{m}$ is the adsorption capacity under monolayer adsorption; $k_{F}$ is the Freundlich relative capacity coefficient; $n$ is the Freundlich empirical adsorption intensity coefficient.

The concentrations of E2 and EE2 were determined by an ACQUITY UHPLC system (Waters Co., Milford, USA) with fluorescence detector. Compounds were separated by a C18 reversed-phase column $(2.1 \times 50 \mathrm{~mm}$, particle size of $1.7 \mu \mathrm{m})$. Mobile phase was $50 \%$ water and $50 \%$ acetonitrile. Injection volume was $10 \mu \mathrm{L}$ and flow rate was set at $0.2 \mathrm{~mL} \mathrm{~min}^{-1}$. E2 and EE2 were detected at wavelength of $310 \mathrm{~nm}$ with excitation wavelength of $280 \mathrm{~nm}$.

\section{Results and Discussion}

PE and PP particles with sphere structure were generally smoother than PVC particles which showed folded structure and rough surface to possibly supply more adsorption sites (Fig. 1). Adsorption of EDCs by microplastics was related with target compounds and plastic materials. The distribution coefficients of $\mathrm{E} 2$ on microplastics followed the order of $\mathrm{PP}>\mathrm{PE}>\mathrm{PVC}(4.7 \mathrm{~mm})$ while those of EE2 followed the order of $\mathrm{PP}<\mathrm{PE}<\mathrm{PVC}$ (Fig. 1a). In general, the larger distribution coefficient is, the stronger adsorption capacity will be. Therefore, the adsorption capacity of PVC for EE2 might be the highest among three types of microplastics, similar with the results reported by Guo et al. (2018). PVC, widely used in the world, showed significantly high adsorption potential for EE2 to be selected as target microplastic for the following studies. The particle size of microplastics played an important role in the adsorption of EDCs. Microplastics with smaller particle size significantly possessed higher distribution coefficient for target EDCs (Fig. 1b), which could be explained by reason that smaller microplastic particles possessed larger specific surface area for easier adsorption of the pollutants. Distribution coefficient of E2/EE2 on PVC $(0.11 \mathrm{~mm})$ microplastics was $24.2 / 4.51$ times that on PVC microplastics (4.7 mm). Distribution coefficient of E2/EE2 

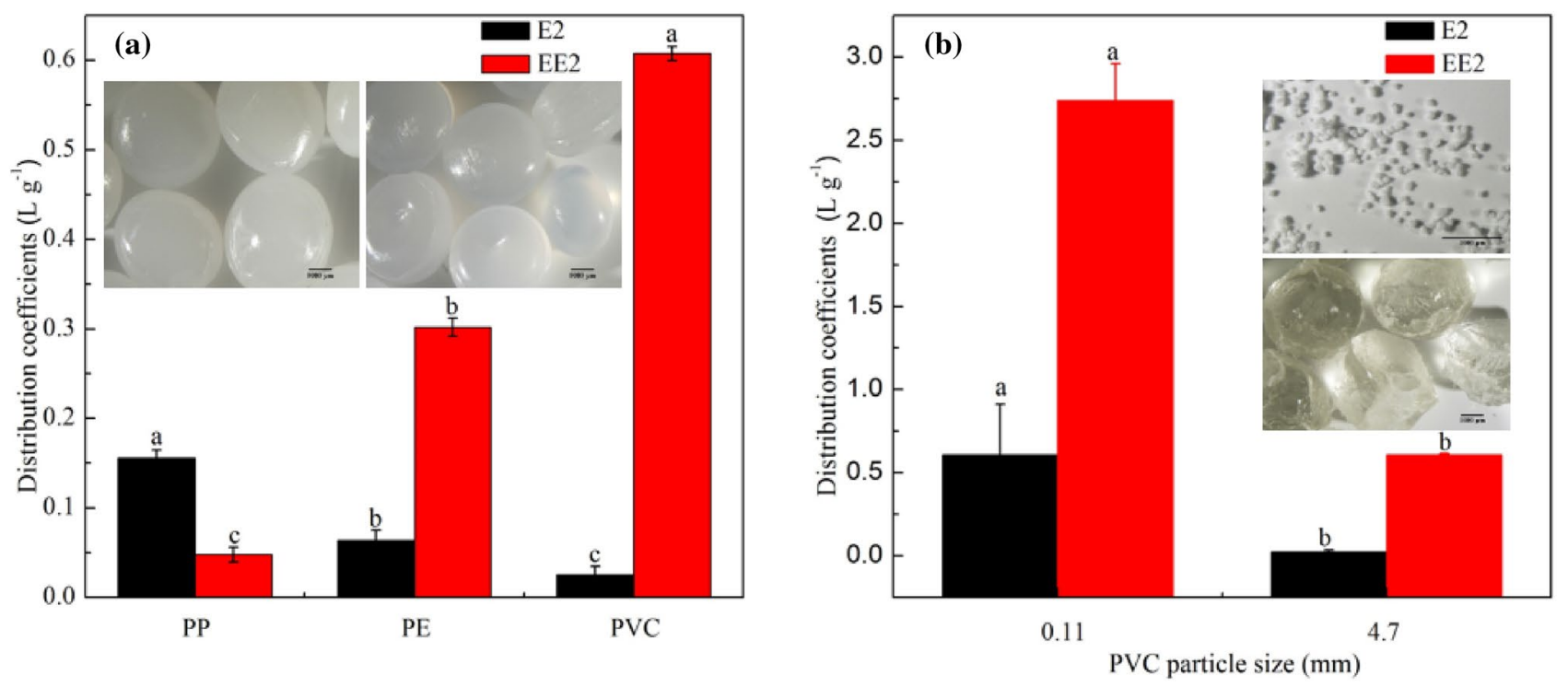

Fig. 1 Effects of microplastic source materials (a) and PVC particle size (b) on adsorption of E2 and EE2. Stereomicroscopic image in left/right of (a) was for PP/PE while that in top/bottom of (b) was for PVC with average size of $0.11 / 4.7 \mathrm{~mm}(\mathrm{n}=3)$

on PVC $(0.11 \mathrm{~mm})$ microplastics reached $0.61 / 2.74 \mathrm{~L} \mathrm{~g}^{-1}$, showing that physico-chemical properties of EDCs possibly had impacts on their adsorption on microplastics.

Effects of different factors on adsorption of E2/EE2 by microplastics (PVC with particle size of $0.11 \mathrm{~mm}$ ) were investigated (Fig. 2). Salinity had different effects on adsorption of E2 and EE2 on microplastics (Fig. 2a). Adsorption capacity of $\mathrm{E} 2$ by microplastics increased with salinity and reached $33.56 \mu \mathrm{g} \mathrm{g}^{-1}$ at salinity of $32 \mathrm{~g} \mathrm{~L}^{-1}$, almost 3.3 times that at $16 \mathrm{~g} \mathrm{~L}^{-1}$. Adsorption capacity of EE2 almost unchanged when salinity was lower than $24 \mathrm{~g} \mathrm{~L}^{-1}$ while that significantly increased to $40.16 \mu \mathrm{g} \mathrm{g}^{-1}$ at salinity of $32 \mathrm{~g} \mathrm{~L}^{-1}$. High salinity could enhance the electrostatic attraction between pollutants and microplastics. Moreover, the solubility of pollutants decreased as salinity increased to prompt more pollutant particles to distribute on the microplastics (Wu et al. 2003; Lu et al. 2017). The effect of $\mathrm{pH}$ on adsorption of E2 and EE2 by microplastics was similar. Adsorption capacity of E2/EE2 firstly increased to reach the highest at $\mathrm{pH}$ of 8.0 and then decreased when $\mathrm{pH}$ further increased (Fig. 2b). This variation might be caused by different speciation of E2/EE2 in seawater at various $\mathrm{pH}$ and the change of the surface charge of microplastics. HA had strong effects on adsorption of E2/EE2 by microplastics (Fig. 2c). Adsorption capacity of E2/EE2 rapidly increased when HA concentrations increased from 0 to $10 \mathrm{mg} \mathrm{L}^{-1}$ while it slowly increased when HA concentrations further increased to $20 \mathrm{mg} \mathrm{L}^{-1}$ (Fig. 2c). HA was generally reported to decrease adsorption of EDCs on adsorbent due to formation of soluble EDCs-HA complexes (Jiang et al. 2016). The opposite results of this study might be caused by speciation of HA and EDCs in seawater as well as interaction between HA, EDCs, and microplastic particles. Adsorption capacity of E2/EE2 on microplastics increased with initial concentrations of target EDCs (Fig. 2d), similar with adsorption pattern of other pollutants on biochar (Wang et al. 2015). Higher initial concentrations of target compounds increased possible contact between pollutant particles and adsorbent particles to enhance the adsorption capacity. Salinity, HA concentration, and initial E2/EE2 concentration all showed a strong correlation with the adsorption amount of target hormones.

The adsorption kinetics of E2 and EE2 on PVC microplastics were investigated (Fig. 2e, f). Adsorption of E2 and EE2 on microplastics showed the similar pattern and reached equilibrium after $48 \mathrm{~h}$. Adsorption capacity of E2/EE2 rapidly reached 33.56/40.16 $\mu \mathrm{g} \mathrm{g}^{-1}$ within $48 \mathrm{~h}$ and then slowly increased to reach $40.00 / 43.99 \mathrm{\mu g} \mathrm{g}^{-1}$ at $96 \mathrm{~h}$. Interestingly, both pseudo-first-order model and pseudo-second-order kinetics could well fit adsorption data of $\mathrm{E} 2$ with $\mathrm{R}^{2}>0.930$ and pseudo-second-order model was slightly better with $\mathrm{R}^{2}=0.948$ (Fig. 2e). The pseudo-second-order kinetics rate constant $k_{2}$ was $0.0011 \mathrm{~g} \mathrm{\mu g}^{-1} \mathrm{~h}^{-1}$ and the calculated saturation adsorption of E2 reached $47.11 \mu \mathrm{g} \mathrm{g}^{-1}$. Pseudo-firstorder model was slightly better than pseudo-second-order kinetics to fit the adsorption data of EE2 with $\mathrm{R}^{2}=0.943$ (Fig. 2f). The rate constant $k_{l}$ was $0.0477 \mathrm{~h}^{-1}$ and the calculated saturation adsorption of EE2 was $43.88 \mu \mathrm{g} \mathrm{g}^{-1}$. Generally, the physical process may be the rate-limiting step of adsorption if pseudo-first-order kinetics can well explain the adsorption data while the chemisorption may be the main adsorption mechanism if pseudo-second-order model well 

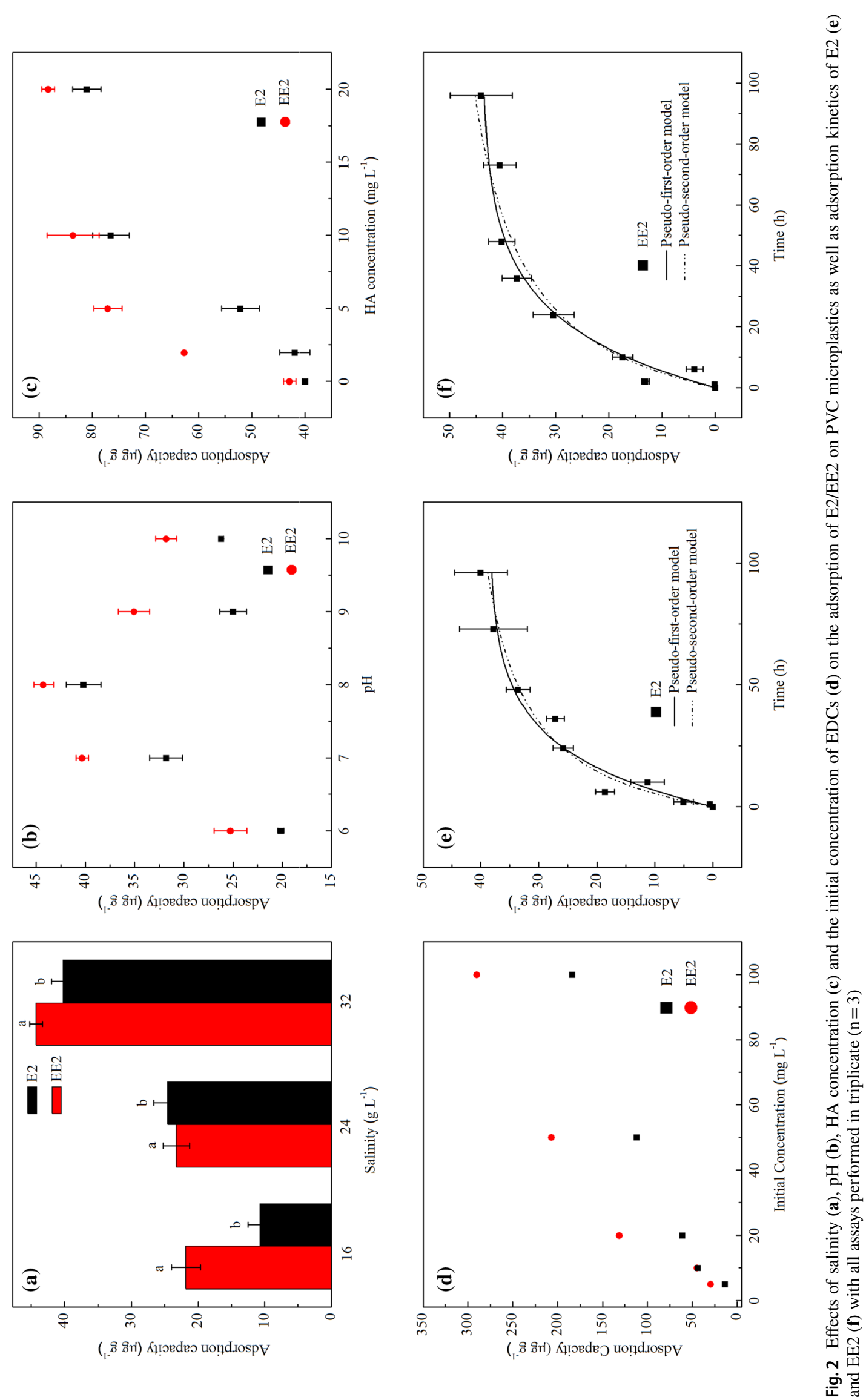
fits the adsorption data (Jiang et al. 2016; Wu et al. 2019). Two models could well explain adsorption of E2/EE2 on PVC microplastics, suggesting that both chemical and physical adsorption contributed to interfacial behaviors of target EDCs on microplastics in seawater.

Temperature was significantly related to adsorption of E2 \& EE2 and it might become the most important factor influencing the adsorption of E2 and EE2 on microplastics. Langmuir and Freundlich models were employed to investigate the adsorption isotherm of E2 and EE2 on microplastics (Fig. 3). Freundlich isotherm was better to fit the adsorption data of E2 than Langmuir model (Fig. 3a), suggesting that adsorption of E2 on PVC microplastics took place on heterogeneous surface (Freundlich 1906; Jiang et al. 2016; Wang et al. 2015). Freundlich parameter $n$ was larger than 1.00 for all treatments of E2, suggesting that E2 was easily adsorbed on PVC microplastics (Wang et al. 2015).

Langmuir isotherm yielded better fitting results for EE2 than Freundlich model (Fig. 3b), suggesting that EE2 adsorption on PVC microplastics occurred at specific homogeneous adsorbent sites (Langmuir 1918; Lu et al. 2017). The possible maximal adsorption capacity of EE2 on PVC microplastics reached $1654.36 \mu \mathrm{g} \mathrm{g}^{-1}$ estimated by Langmuir model, showing the potential risks of microplastics in seawater. In addition, adsorption capacity of E2/EE2 decreased as temperature increase, similar with previous report (Jiang et al. 2016; Wu et al. 2019). Adsorption free energy $(\Delta G)$ for $E 2$ ranged from -7.25 to $-1.31 \mathrm{~kJ} \mathrm{~mol}^{-1}$ while it for EE2 varied -7.29 to $-0.41 \mathrm{~kJ} \mathrm{~mol}^{-1}$, suggesting that adsorption of target EDCs on PVC microplastics was a spontaneous process (Lu et al. 2017). Moreover, $\Delta \mathrm{G}$ increased with temperature to show that lower temperature would be good for adsorption of target EDCs, which could also explain the effects of temperature on adsorption capacity of target EDCs by PVC microplastics. Thermodynamic parameters including adsorption entropy $(\Delta \mathrm{S})$ and enthalpy $(\Delta \mathrm{H})$ were also calculated. $\Delta \mathrm{H}$ for E2 was $-89.76 \mathrm{~kJ} \mathrm{~mol}^{-1}$ while it for EE2 was $-102.35 \mathrm{~kJ} \mathrm{~mol}^{-1}$, implying that adsorption of target EDCs on PVC microplastics was exothermic process (Wu et al. 2019). $\Delta S$ for $E 2$ was $-0.292 \mathrm{~kJ} \mathrm{~mol}^{-1}$ while it for EE2 was $-0.335 \mathrm{~kJ} \mathrm{~mol}^{-1}$, much lower than that of other organic pollutants adsorbed on microplastics (Wu et al. 2019).

Desorption of E2 and EE2 from PVC microplastics was also discussed (Fig. 4). Both pseudo-first-order and pseudosecond-order kinetics could yield good fitting results for desorption data of E2 $\left(R^{2}=0.992\right)$ and EE2 $\left(R^{2}=0.987\right)$.

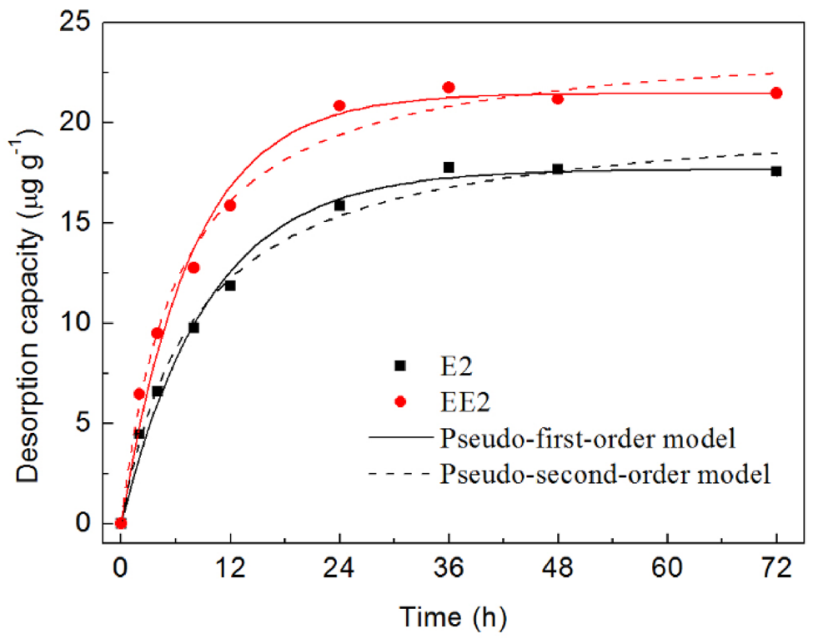

Fig. 4 Desorption kinetics of E2 and EE2 on PVC microplastics $(\mathrm{n}=3)$
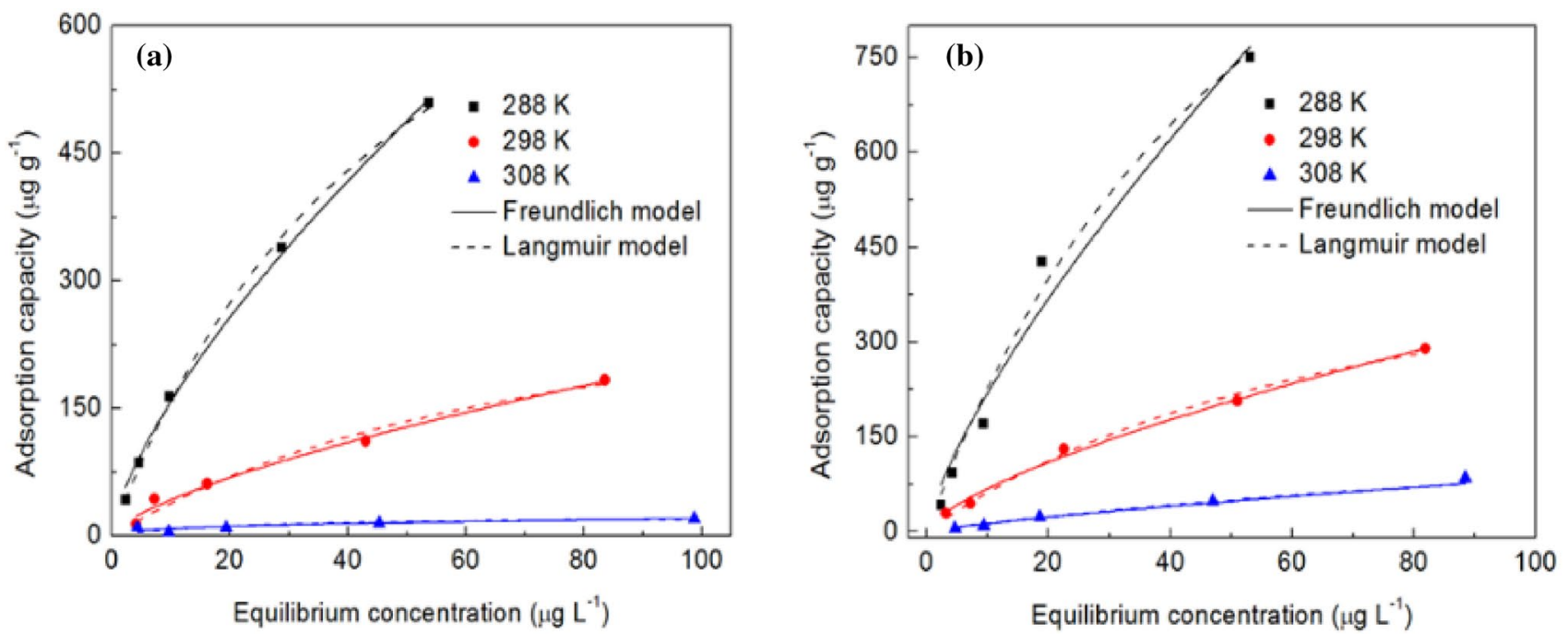

Fig. 3 Adsorption isotherms of E2 (a) and EE2 (b) on PVC microplastics ( $n=3$ ) 
Desorption reached equilibrium at $36 \mathrm{~h}$ and the highest desorption capacity of E2/EE2 was $17.58 / 21.46 \mu \mathrm{g} \mathrm{g}^{-1}$. The maximal desorption capacity of E2 was nearly $44.0 \%$ of its corresponding adsorption capacity while the highest desorption capacity of EE2 reached nearly $48.8 \%$ of its adsorption capacity. The adsorption/desorption capacity of target hormone on microplastics was positively related to its hydrophobicity, which was similar with the previous study on persistent organic pollutants (León et al. 2019). Relatively high desorption capacity of target EDCs on PVC microplastics might exert the potential risks to the aquatic ecosystems since the desorbed contaminants might re-enter the seawater, sediments, and organisms.

This study provided new insights on adsorption and desorption behaviors of E2 and EE2 on microplastics in seawater. Material and size of microplastics affected the adsorption of E2 and EE2. The increase in salinity, HA concentration, and initial E2/EE2 concentration could increase the adsorption of E2/EE2 on microplastics. Adsorption capacity of E2/ $\mathrm{EE} 2$ generally reached the highest at $\mathrm{pH}$ of 8.0. Desorption capacity of E2/EE2 accounted for over $40 \%$ of adsorption capacity to pose potential risks. Factors affecting desorption of target hormones on microplastics in seawater need to be further discussed in the future.

Acknowledgements This work was supported by National Natural Science Foundation of China (No. 41671319), Taishan Scholar Program of Shandong Province (No. tsqn201812116), Two-Hundred Talents Plan of Yantai (No. Y739011021), One Hundred Talents Program of Chinese Academy of Sciences (No. Y629041021), and Key Research Program of Frontier Sciences of CAS (No. QYZDJ-SSW-DQC015). The authors would like to thank the editor and reviewers for their valuable suggestions and comments on the manuscript.

\section{References}

Andrady AL (2011) Microplastics in the marine environment. Mar Pollut Bull 62:1596-1605

Barboza LGA, Gimenez BCG (2015) Microplastics in the marine environment: current trends and future perspectives. Mar Pollut Bull 97:5-12

Brian JV, Harris CA, Scholze M, Kortenkamp A, Booy P, Lamoree M, Pojana G, Jonkers N, Marcomini A, Sumpter JP (2007) Evidence of estrogenic mixture effects on the reproductive performance of fish. Environ Sci Technol 41(1):337-344

Freundlich H (1906) Über die Adsorption in Lösungen. W. Engelmann, Leipzig
Giulivo M, Lopez de Alda M, Capri E, Barceló D (2016) Human exposure to endocrine disrupting compounds: their role in reproductive systems, metabolic syndrome and breast cancer. A review. Environ Res 151:251-264

Guo X, Pang J, Chen S, Jia H (2018) Sorption properties of tylosin on four different microplastics. Chemosphere 209:240-245

Jiang L, Liu Y, Zeng G, Xiao F, Hu X, Hu X, Wang H, Li T, Zhou L, Tan X (2016) Removal of 17b-estradiol by few-layered graphene oxide nanosheets from aqueous solutions: external influence and adsorption mechanism. Chem Eng J 284:93-102

Langmuir I (1918) The adsorption of gases on plane surfaces of glass, mica and platinum. J Am Chem Soc 40:1361-1403

León VM, García-Agüera I, Moltó V, Fernández-González V, LlorcaPérez L, Andrade JM, Muniategui-Lorenzo S, Campillo JA (2019) PAHs, pesticides, personal care products and plastic additives in plastic debris from Spanish Mediterranean beaches. Sci Total Environ 670:672-684

Lu J, Zhang C, Wu J, Luo Y (2017) Adsorptive removal of bisphenol A using n-doped biochar made of Ulva prolifera. Water Air Soil Pollut 228:327

Mato Y, Isobe T, Takada H, Kanehiro H, Ohtake C, Kaminuma T (2001) Plastic resin pellets as a transport medium for toxic chemicals in the marine environment. Environ Sci Technol 35(2):318-324

Meng F, Yuan G, Wei J, Bi D, Wang H (2017) Leonardite-derived humic substances are great adsorbents for cadmium. Environ Sci Pollut Res 24:23006-23014

O'Brine T, Thompson RC (2010) Degradation of plastic carrier bags in the marine environment. Mar Pollut Bull 60(12):2279-2283

Ogata Y, Takada H, Mizukawa K, Hirai H, Iwasa S, Endo S, Mato Y, Saha M, Okuda K, Nakashima A, Murakami M, Zurcher N, Booyatumanondo R, Zakaria MP, le Dung Q, Gordon M, Miguez C, Suzuki S, Moore C, Karapanagioti HK, Weerts S, McClurg T, Burres E, Smith W, Van Velkenburg M, Lang JS, Lang RC, Laursen D, Danner B, Stewardson N, Thompson RC (2009) International pellet watch: global monitoring of persistent organic pollutants (POPs) in coastal waters 1 Initial phase data on PCBs, DDTs, and HCHs. Mar Pollut Bull 58(10):1437-1446

Wang Y, Lu J, Wu J, Liu Q, Zhang H, Jin S (2015) Adsorptive removal of fluoroquinolone antibiotics using bamboo biochar. Sustainability 7:12947-12957

Wu P, Yang G-P, Zhao X-K (2003) Sorption behavior of 2,4-dichlorophenol on marine sediment. J Colloid Interf Sci 265(2):251-256

Wu P, Cai Z, Jin H, Tang Y (2019) Adsorption mechanisms of five bisphenol analogues on PVC microplastics. Sci Total Environ 650:671-678

Ying G-G, Kookana RS, Ru Y-J (2002) Occurrence and fate of hormone steroids in the environment. Environ Int 28(6):545-551

Zhang H, Wang J, Zhou B, Zhou Y, Dai Z, Zhou Q, Chriestie P, Luo Y (2018) Enhanced adsorption of oxytetracycline to weathered micro-plastic polystyrene: kinetics, isotherms and influencing factors. Environ Pollut 243:1550-1557 